\title{
Moderate alcohol consumption is associated with improved insulin sensitivity, reduced basal insulin secretion rate and lower fasting glucagon concentration in healthy women
}

\author{
F. Bonnet • E. Disse • M. Laville • A. Mari • K. Hojlund • \\ C. H. Anderwald • P. Piatti • B. Balkau • \\ for the RISC Study Group
}

Received: 10 June 2012 / Accepted: 31 July 2012 /Published online: 31 August 2012

(C) Springer-Verlag 2012

\begin{abstract}
Aims/hypothesis Moderate alcohol consumption is associated with a reduced risk of type 2 diabetes with a stronger effect in women. As the underlying mechanisms remain poorly characterised, we investigated its relationship with insulin resistance, insulin secretion, clearance of insulin and glucagon concentration.
\end{abstract}

The list of RISC investigators is presented in the electronic supplementary material (ESM).

Electronic supplementary material The online version of this article (doi:10.1007/s00125-012-2701-3) contains peer-reviewed but unedited supplementary material, which is available to authorised users.

F. Bonnet $(\triangle)$

Service Endocrinologie-Diabétologie, CHU Rennes,

Université Rennes 1,

Inserm UMR 991,

Rennes, France

e-mail: fbonnet29@orange.fr

E. Disse $\cdot$ M. Laville

Centre de Recherche en Nutrition Humaine, CRNH Rhône-Alpes,

Inserm U1060-INRA 1235

Lyon, France

\section{A. Mari}

Institute of Biomedical Engineering,

Padua, Italy

\section{K. Hojlund}

Department of Endocrinology, Odense University Hospital,

Odense, Denmark

C. H. Anderwald

Department of Internal Medicine III,

Medical University of Vienna,

Vienna, Austria
Methods One-thousand two-hundred and seventy-six non-diabetic individuals from the RISC (relationship between insulin sensitivity and cardiovascular disease) study without high alcohol consumption were studied; all had a euglycaemic-hyperinsulinaemic clamp and an OGTT with assessment of insulin sensitivity, secretion and clearance.

C. H. Anderwald

Medical Direction, Specialized Hospital Complex Agathenhof, Micheldorf, Austria

C. H. Anderwald

Metabolic Unit, Istituto di Ingegneria Biomedica-Consiglio

Nazionale delle Ricerche (ISIB-CNR),

Padova, Italy

P. Piatti

San Raffaele Scientific Institute, Internal Medicine Department, and Metabolic and Cardiovascular Science Division,

Milan, Italy

B. Balkau

Inserm, CESP Centre for Research in Epidemiology and

Population Health, U1018, Epidemiology of diabetes,

obesity and chronic kidney disease over the lifecourse and

determinants of early nutrition,

Villejuif, France

B. Balkau

University Paris Sud 11,

UMRS 1018

Villejuif, France 
Results Alcohol consumption was positively associated with insulin sensitivity in women $\left(\beta=0.15, p_{\text {trend }}=0.005\right)$ and in men $\left(\beta=0.07, p_{\text {trend }}=0.07\right)$ after controlling for age, centre, waist, smoking and physical activity. In women, this association persisted after adjustment for adiponectin but was attenuated after controlling for HDL-cholesterol, suggesting that part of the protection is related to a higher HDL-cholesterol concentration. Higher alcohol consumption was associated with lower basal insulin secretion in women only $\left(\beta=-0.10, p_{\text {trend }}=0.004\right)$ and this association persisted after adjustment for insulin sensitivity. In men, increasing alcohol consumption was associated with enhanced insulin clearance and increased fasting NEFA concentrations, independently of insulin sensitivity. Fasting glucagon decreased with increasing alcohol in women only (abstainers $9.2 \pm 4.4 ;<28 \mathrm{~g} /$ week $8.6 \pm 4.0 ; 28-64 \mathrm{~g} /$ week $8.1 \pm 3.7 ;>64 \mathrm{~g} /$ week $7.5 \pm 3.1 \mathrm{pmol} / 1 ; p_{\text {trend }}=0.01$ ).

Conclusions/interpretation Light-to-moderate alcohol consumption was associated in healthy women with enhanced insulin sensitivity, reduced basal insulin secretion rate and lower fasting plasma glucagon concentration, providing consistent mechanisms for the reduced risk of diabetes.

Keywords Alcohol · Glucagon · Insulin clearance · Insulin resistance $\cdot$ Insulin secretion

\section{Abbreviations \\ GGT Gamma glutamyl transferase \\ IGT Impaired glucose tolerance \\ RISC Relationship between insulin sensitivity and cardiovascular disease}

\section{Introduction}

Epidemiological studies have shown that moderate alcohol consumption is associated with a reduced risk of type 2 diabetes [1-6]. This has been confirmed by a recent metaanalysis of observational studies that reported a U-shaped relationship for both sexes with a risk reduction for a moderate alcohol intake and an increased risk for an intake of over 50-60 g/day [7]. The relationship differed between men and women, with a lower RR in women than men for moderate alcohol intake, compared with alcohol abstainers.

The underlying mechanisms behind this reduction in risk with moderate alcohol intake remain poorly understood. It has been proposed that this risk reduction might be explained by improved insulin sensitivity but this has not been assessed by the method of reference, the hyperinsulinaemic-euglycaemic clamp. Furthermore, the relationship between alcohol consumption and both glucagon concentration and insulin secretion has not been previously addressed.
The aim of the present study is to determine the relationship between moderate alcohol intake and both insulin sensitivity (assessed by the hyperinsulinaemic-euglycaemic clamp) and insulin secretion in a large cohort of healthy men and women participating in the RISC study (Relationship between Insulin Sensitivity and Cardiovascular disease study) $[8,9]$. We performed a sex-specific analysis, as mean alcohol intake was significantly lower in women and also to determine possible sex differences in the association between alcohol consumption and glucose metabolism, as seen in the meta-analysis [7]. Furthermore, we aimed to explore the potential confounding role of adiponectin and HDL-cholesterol in mediating the relationship between alcohol intake and glucose metabolism.

\section{Methods}

Study population RISC is a prospective observational cohort study whose rationale and methodology have been published, as well as the characteristics of the individuals recruited $[8,9]$. Clinically healthy men and women, aged 30-60 years, were recruited by advertisement, from the local populations of 19 centres in 14 European countries. Initial exclusion criteria were: treatment for obesity, hypertension, lipid disorders or diabetes, pregnancy, cardiovascular or chronic lung disease, weight change $\geq 5 \mathrm{~kg}$ in the last 6 months, cancer (in the last 5 years) and renal failure. Exclusion criteria after screening were: arterial $\mathrm{BP} \geq 140$ / $90 \mathrm{mmHg}$, fasting plasma glucose $\geq 7.0 \mathrm{mmol} / \mathrm{l}, 2 \mathrm{~h}$ plasma glucose (following a $75 \mathrm{~g}$ OGTT) $\geq 11.0 \mathrm{mmol} / \mathrm{l}$, total serum cholesterol $\geq 7.8 \mathrm{mmol} / 1$, serum triacylglycerol $\geq 4.6$ $\mathrm{mmol} / \mathrm{l}$ and ECG abnormalities. In the present analysis, we included all the participants who were not ruled out by these criteria, whose clamp study passed the quality control check and for whom data on HDL-cholesterol and alcohol intake were available $(n=1,289)$. We also excluded those (nine men and four women) with a high alcohol consumption (defined as $>350 \mathrm{~g} /$ week for men and $>280 \mathrm{~g} /$ week for women), as the purpose of the study was to investigate the metabolic effects of lightto-moderate alcohol consumption. Therefore, we studied a total of 1,276 individuals.

At 3 years 563 women and 461 men attended a second OGTT. Impaired glucose tolerance (IGT), defined as fasting glucose $<7.0 \mathrm{mmol} / 1$ and $2 \mathrm{~h}$ glucose $\geq 7.8 \mathrm{mmol} / \mathrm{l}$ but $<11 \mathrm{mmol} / 1$ or type 2 diabetes, was present in $58 \mathrm{men}$ and 78 women.

Height was measured, and body weight and per cent body fat were evaluated by the TANITA bioimpedance balance (Tanita International Division, Arlington Heights, IL, USA). BMI was calculated and obesity was defined as BMI $\geq 30 \mathrm{~kg} / \mathrm{m}^{2}$. 
Ethics Committee approval was obtained by each recruiting centre. Participants were given detailed written information on the study as well as an oral explanation, and they all signed a consent form.

Lifestyle and alcohol consumption Alcohol consumption was assessed using a standardised semiquantitative food-frequency questionnaire. For each beverage type (beer, red and white wine, port, vermouth, hard liquor), participants reported their usual number of glasses and the glass size (small, medium or large, according to photographs on the questionnaire). The ten frequency response categories ranged from 'never' to ' 40 or more times per week'. The consumption was quantified in grams of pure alcohol per week and then stratified into four categories: abstainers; by tertiles of alcohol intake, for men, $<57,57-130,>130 \mathrm{~g} /$ week and for women, $<28,28-64,>64$ g/week.

Information on physical activity was collected with the 7day International Physical Activity Questionnaire (IPAQ), a previously validated assessment tool for international studies, that provides a comprehensive evaluation of daily physical-activity habits [10].

OGTT OGTT was performed after a fasting period of at least $10 \mathrm{~h}$. Blood samples were taken at fasting and 30 , 60, 90 and $120 \mathrm{~min}$ into the OGTT, together with samples for central analysis of routine blood chemistry. Blood collected during the studies was separated into plasma and serum, portioned and stored at $-20^{\circ} \mathrm{C}$ for glucose and insulin and $-80^{\circ} \mathrm{C}$ for lipid analysis.

Glucose concentrations were measured by the glucose oxidase technique. Plasma insulin and C-peptide were measured by a two-site time-resolved fluoroimmunoassay (AutoDELFIA Insulin kit; Wallac, Turku, Finland) using monoclonal antibodies, with the following assay characteristics (for insulin and C-peptide, respectively): sensitivity 12 and $5 \mathrm{pmol} / \mathrm{l}$, within-assay variation 5 and $5 \%$ and between-assay variation 5 and $3.5 \%$. Liver enzymes were centrally assayed on a Dade-Behring Dimension RXL Autoanalyser (Cambridge, UK). Measurement of plasma NEFA concentration was carried out using a Randox enzymatic kit (Randox Laboratories Limited, Crumlin, UK). The CV was less than $5 \%$. Serum adiponectin was determined by an in-house time-resolved immunofluorometric assay (TRIFMA), based on two antibodies and recombinant human adiponectin, that measures total circulating adiponectin (including high- and low-molecular-mass isoforms) (R \& D Systems, Abingdon, UK) [11]. All standards and unknown samples were analysed in duplicate, with the exception of non-specific binding, which was analysed in quadruplicate. The intra-assay $\mathrm{CV}$ was $<5 \%$ and the inter-assay $\mathrm{CV}$ was $<10 \%$. The samples for glucagon assessment were collected in a tube containing EDTA plus a protease inhibitor (aprotinin) and repeated freeze-thaw of these samples was avoided. Glucagon concentration was measured in a centralised laboratory in Odense, Denmark by an immunoassay method that is highly specific for the free $\mathrm{C}$-terminus of the molecule, and therefore specific for pancreatic glucagon, with the following assay characteristics: normal range (5$20 \mathrm{pmol} / \mathrm{l})$ sensitivity $<1 \mathrm{pmol} / \mathrm{l}$, within-assay $\mathrm{CV}<5 \%$ at 20 pmol/l, between-assay CV $<12 \%$ [11].

Insulin sensitivity On a separate day within 1 month of the OGTT, a hyperinsulinaemic-euglycaemic clamp was performed. Exogenous insulin was administered as a primed continuous infusion at a rate of $240 \mathrm{pmol} \mathrm{min} \mathrm{m}^{-1} \mathrm{~m}^{-2}$ simultaneously with a variable $20 \%$ dextrose infusion adjusted every 5-10 min to maintain the plasma glucose concentration within $0.8 \mathrm{mmol} / 1( \pm 15 \%)$ of the target glucose level $(4.5-5.5 \mathrm{mmol} / \mathrm{l})$. The clamp procedure was standardised across centres; the data from each clamp study were transferred to the coordinating centre where they underwent quality-control scrutiny according to pre-set criteria.

Insulin sensitivity was expressed as the ratio of the $M$ value during the final $40 \mathrm{~min}$ of the $2 \mathrm{~h}$ clamp, to the mean plasma insulin concentration measured during the same interval $(M / I)$, normalised to fat-free mass and expressed in units of $\mu \mathrm{molmin}^{-1}$ (kg fat free mass $)^{-1}(\mathrm{nmol} / 1)^{-1}$

Insulin secretion Beta cell function was assessed from the OGTT using a model describing the relationship between insulin secretion (calculated from C-peptide using the method of van Cauter et al [12]) and glucose concentration, previously described in detail [13-15]. From the model-estimated beta cell dose-response, relating insulin secretion (in pmol $\mathrm{min}^{-1} \mathrm{~m}^{-2}$ ) to glucose concentration, insulin secretion at $5 \mathrm{mmol} / \mathrm{l}$ glucose (the average basal glucose in the participants with normal glucose tolerance) was determined. This variable represents insulin secretion in basal conditions if basal glucose was $5 \mathrm{mmol} / \mathrm{l}$ in each individual. Basal and total (integral during the OGTT, in $\mathrm{nmol} / \mathrm{m}^{2}$ ) insulin secretion was also determined using the model.

Insulin clearance Peripheral insulin clearance during the clamp (in $1 \mathrm{~min}^{-1} \mathrm{~m}^{-2}$ ) was calculated as the ratio between insulin infusion rate and steady-state insulin concentration in the last $40 \mathrm{~min}$ of the clamp.

The endogenous 'pre-hepatic' clearance in basal conditions was defined as: (basal insulin secretion)/(basal insulin concentration) where basal insulin secretion is calculated from the beta cell modelling analysis. This clearance value is directly dependent on hepatic insulin extraction. The endogeneous 'pre-hepatic' insulin clearance during the OGTT (in $1 \mathrm{~min}^{-1} \mathrm{~m}^{-2}$ ) was defined as the ratio of mean insulin secretion and mean insulin concentration. 
Statistical analysis The data are expressed as mean $\pm \mathrm{SD}$ or as median (interquartile range) for variables with a skewed distribution, and categorical data as percentages. Variables that were not symmetrically distributed were log transformed before analyses. Data were analysed for men and women separately.

The mean values of the participant characteristics were compared across categories of alcohol intake by ANOVA. Spearman correlation coefficients were first assessed between alcohol consumption, as expressed in $\mathrm{g} /$ week as a continuous variable, and insulin sensitivity.

The relationships between alcohol intake categories, and insulin sensitivity, insulin secretion, insulin clearance and glucagon were assessed by linear regression analysis with adjustment for age, recruitment centre, physical activity, current smoking and waist circumference. Trend tests were determined across the categories of alcohol intake. Next, HDL-cholesterol, triacylglycerol and adiponectin levels were added into the regression model. We also tested for an interaction between BMI $\leq 25$ and $>25 \mathrm{~kg} / \mathrm{m}^{2}$ with alcohol consumption categories on insulin sensitivity. Logistic regression analysis was used to assess the relationship between alcohol consumption and the risk of impaired glucose tolerance or type 2 diabetes at year3, after adjustment for age and waist circumference. Statistical analyses used StatView for Windows (version 5.0; SAS Institute, Cary, NC, USA) and SAS version 9.2.

\section{Results}

The percentage of abstainers was higher in women $(n=172$, $24.4 \%)$ than in men $(n=72,12.6 \%)$. When excluding the abstainers, the median alcohol intake was $84 \mathrm{~g} /$ week (range $7.5-350 \mathrm{~g} /$ week) in men and $42 \mathrm{~g} /$ week in women (range $7.5-270 \mathrm{~g} /$ week).

In women only, moderate alcohol consumption was associated with a lower BMI and waist circumference and with a higher prevalence of current smoking (Tables 1,2). Fasting and $2 \mathrm{~h}$ glycaemia did not differ according to alcohol consumption categories in either men or women.

In both men and women, there was a graded increase in plasma HDL-cholesterol and gamma glutamyl transferase (GGT) across categories of alcohol intake (Tables 1, 2). Adiponectin concentrations did not significantly differ according to alcohol consumption categories in either men or women, but there was a trend for a positive relation in women $(p=0.08)$.

Insulin sensitivity In univariate analysis, alcohol consumption, expressed as a continuous variable, was positively correlated with insulin sensitivity in women $\left(r_{\text {Spearman }}=\right.$ $0.16, p=0.0001)$ but not in men $\left(r_{\text {Spearman }}=0.05, p=0.20\right)$.
When alcohol was used as a categorical variable the results were very similar, with a significant association in women ( $p=0.005)$ but not in men $(p=0.07)$, after controlling for age, recruitment centre, waist, current smoking and physical activity (Fig. 1). This association persisted after excluding women who were alcohol abstainers from the analysis. The significance of the relation between alcohol consumption categories and insulin sensitivity was only borderline after controlling for HDL-cholesterol concentration in women $(p=0.06)$, and there was no relation in men $(p=0.24)$. Further adjustment for adiponectin, triacylglycerol or GGT levels did not alter the significant association between alcohol consumption and the $M / I$ value in women. In men, the relationship between alcohol intake and insulin sensitivity was stronger after controlling for GGT levels $(p=0.02)$. There was no significant interaction between BMI $\leq /$ $>25 \mathrm{~kg} / \mathrm{m}^{2}$ and alcohol consumption on insulin sensitivity in either sex.

Insulin secretion Basal insulin secretion decreased across alcohol consumption categories in women but not in men, after adjusting for age, recruitment centre, smoking, physical activity and waist circumference (Fig. 2). The significant inverse association between increased alcohol consumption and basal insulin secretion in women persisted after controlling for insulin sensitivity $(\log M / I)\left(p_{\text {trend }}=0.017\right)$ or for HDL-cholesterol, albeit being attenuated after taking this variable into account ( $\left.p_{\text {trend }}=0.03\right)$.

We also quantified basal insulin secretion, at a fixed normal glucose level of $5 \mathrm{mmol} / \mathrm{l}$ glucose, from the beta cell dose-response. There was an inverse association between alcohol intake and insulin secretion at $5 \mathrm{mmol} / \mathrm{lglu}$ cose in women only, with a progressive decrease in insulin secretion rate across alcohol consumption categories (see electronic supplementary material [ESM] Fig. 1). This association persisted after controlling for the $M / I$ value, suggesting an effect independent of changes in insulin sensitivity. Total insulin secretion during the OGTT did not differ according to the alcohol intake categories, in either sex (ESM Fig. 2).

NEFA concentration In both men and women, there was a significant increase in NEFA levels across alcohol intake categories (Tables 1 and 2). This association persisted after controlling for age, recruitment centre, waist, current smoking and physical activity in men $\left(p_{\text {trend }}=0.0003\right)$ but not in women $\left(p_{\text {trend }}=0.32\right)$.

Insulin clearance Alcohol consumption, expressed in categories, was significantly associated with an increased peripheral clearance of insulin during the clamp and enhanced endogenous 'pre-hepatic' clearance of insulin in both basal 
Table 1 Baseline characteristics according to categories of alcohol consumption in men. The RISC study

\begin{tabular}{|c|c|c|c|c|c|}
\hline \multirow[t]{2}{*}{ Characteristic } & \multicolumn{4}{|c|}{ Alcohol consumption (g/week) } & \multirow[t]{2}{*}{$p_{A N O V A}$} \\
\hline & Abstainers $(n=72)$ & $<57(n=169)$ & $57-130(n=167)$ & $>130(n=162)$ & \\
\hline Age (years) & $41.2 \pm 7.9$ & $43.5 \pm 8.4$ & $43.5 \pm 8.8$ & $43.6 \pm 8.6$ & 0.20 \\
\hline BMI $\left(\mathrm{kg} / \mathrm{m}^{2}\right)$ & $26.7 \pm 4.2$ & $26.5 \pm 3.3$ & $26.1 \pm 3.8$ & $26.2 \pm 3.1$ & 0.59 \\
\hline Waist circumference $(\mathrm{cm})$ & $93.8 \pm 11.8$ & $93.4 \pm 9.7$ & $93.0 \pm 11.0$ & $93.6 \pm 9.2$ & 0.93 \\
\hline Current smoking (\%) & 25.0 & 25.0 & 28.5 & 31.5 & 0.13 \\
\hline Physically inactive (\%) & 17.6 & 25.6 & 23.6 & 16.6 & 0.38 \\
\hline Systolic blood pressure $(\mathrm{mmHg})$ & $121.3 \pm 10$ & $122.6 \pm 10$ & $121.6 \pm 11$ & $122.8 \pm 11$ & 0.62 \\
\hline Diastolic blood pressure (mmHg) & $75.7 \pm 7$ & $76.9 \pm 7$ & $75.9 \pm 8$ & $76.9 \pm 7$ & 0.43 \\
\hline Fasting glycaemia $(\mathrm{mmol} / \mathrm{l})$ & $5.4 \pm 1.8$ & $5.1 \pm 0.6$ & $5.3 \pm 0.5$ & $5.3 \pm 0.5$ & 0.17 \\
\hline Fasting insulinaemia (pmol/1) & $34.5(38.0)$ & $27.0(22.9)$ & $26.2(21.2)$ & $30.0(25.0)$ & 0.02 \\
\hline Fasting NEFA (mmol/l) & $0.41(0.26)$ & $0.43(0.25)$ & $0.44(0.20)$ & $0.47(0.28)$ & 0.007 \\
\hline Total cholesterol $(\mathrm{mmol} / \mathrm{l})$ & $4.64 \pm 0.9$ & $4.86 \pm 0.9$ & $4.90 \pm 0.8$ & $4.98 \pm 0.9$ & 0.05 \\
\hline LDL-cholesterol (mmol/l) & $2.95 \pm 0.8$ & $3.10 \pm 0.8$ & $3.08 \pm 0.8$ & $3.08 \pm 0.8$ & 0.59 \\
\hline HDL-cholesterol (mmol/l) & $1.14 \pm 0.3$ & $1.24 \pm 0.3$ & $1.24 \pm 0.3$ & $1.26 \pm 0.3$ & 0.05 \\
\hline Triacylglycerol (mmol/l) & $1.04(0.5)$ & $1.08(0.6)$ & $1.08(0.8)$ & $1.16(0.9)$ & 0.13 \\
\hline Adiponectin (mg/l) & $5.6(2.4)$ & $6.0(3.2)$ & $6.3(3.7)$ & $5.9(3.1)$ & 0.19 \\
\hline GGT (U/l) & $26.0(15.0)$ & $25.0(14.5)$ & $25.0(12.7)$ & $28.0(18.0)$ & 0.007 \\
\hline
\end{tabular}

Data are presented as mean $\pm \mathrm{SD}$, median (interquartile range) or $\%$

conditions and during the OGTT in men and women (Tables 3 and 4). The increased insulin clearance across quartiles of alcohol intake in men was independent of insulin sensitivity $(M / I)$ in the multivariable model (Tables 3 and 4).
Glucagon concentration Fasting glucagon levels progressively decreased across alcohol consumption categories in both sexes, but with a significant trend for women only $\left(p_{\text {trend }}=0.01\right.$, Fig. 3). In women, this association remained significant after controlling for insulin sensitivity $(M / I)$ in

Table 2 Baseline characteristics according to categories of alcohol consumption in women. The RISC study

\begin{tabular}{|c|c|c|c|c|c|}
\hline \multirow[t]{2}{*}{ Characteristic } & \multicolumn{4}{|c|}{ Alcohol consumption (g/week) } & \multirow[t]{2}{*}{$p_{A N O V A}$} \\
\hline & Abstainers $(n=172)$ & $<28(n=168)$ & $28-64(n=181)$ & $>64(n=185)$ & \\
\hline Age (years) & $43.4 \pm 8.4$ & $44.1 \pm 8.1$ & $44.5 \pm 8.1$ & $44.8 \pm 8.1$ & 0.41 \\
\hline BMI $\left(\mathrm{kg} / \mathrm{m}^{2}\right)$ & $25.8 \pm 5.1$ & $24.9 \pm 4.2$ & $24.6 \pm 42$ & $24.0 \pm 3.5$ & 0.0007 \\
\hline Waist circumference $(\mathrm{cm})$ & $83.6 \pm 4.3$ & $81.4 \pm 11.6$ & $80.1 \pm 11.3$ & $79.4 \pm .7$ & 0.006 \\
\hline Current smoking (\%) & 20.2 & 22.1 & 28.2 & 28.6 & 0.01 \\
\hline Physically inactive (\%) & 15.3 & 22.4 & 18.4 & 21.5 & 0.28 \\
\hline Systolic blood pressure $(\mathrm{mmHg})$ & $114.9 \pm 13$ & $113.2 \pm 12$ & $112.9 \pm 12$ & $113.3 \pm 13$ & 0.47 \\
\hline Diastolic blood pressure (mmHg) & $73.9 \pm 8$ & $72.5 \pm 8$ & $71.9 \pm 8$ & $72.7 \pm 8$ & 0.10 \\
\hline Fasting glycaemia (mmol/1) & $4.9 \pm 0.6$ & $4.9 \pm 0.6$ & $5.0 \pm 0.5$ & $5.0 \pm 0.5$ & 0.17 \\
\hline Fasting insulinaemia (pmol/1) & $28.0(25.7)$ & $26.0(18.0)$ & $24.0(21.1)$ & $23.4(16.0)$ & 0.19 \\
\hline Fasting NEFA (mmol/1) & $0.60(0.28)$ & $0.54(0.25)$ & $0.53(0.25)$ & $0.59(0.26)$ & 0.03 \\
\hline Total cholesterol (mmol/l) & $4.71 \pm 0.9$ & $4.89 \pm 0.9$ & $4.70 \pm 0.8$ & $4.81 \pm 0.8$ & 0.13 \\
\hline LDL-cholesterol (mmol/l) & $2.82 \pm 0.8$ & $2.91 \pm 0.9$ & $2.70 \pm 0.8$ & $2.68 \pm 0.8$ & 0.03 \\
\hline HDL-cholesterol (mmol/l) & $1.45 \pm 0.3$ & $1.54 \pm 0.3$ & $1.59 \pm 0.4$ & $1.70 \pm 0.4$ & $<0.0001$ \\
\hline Triacylglycerol (mmol/l) & $0.86(0.55)$ & $0.85(0.47)$ & $0.85(0.42)$ & $0.80(0.48)$ & 0.56 \\
\hline Adiponectin (mg/l) & $8.5(5.1)$ & $9.1(5.0)$ & $9.6(5.1)$ & $9.8(4.8)$ & 0.08 \\
\hline GGT (U/1) & $16.0(9.0)$ & $17.0(9.0)$ & $17.0(8.0)$ & $19.0(12.0)$ & $<0.0001$ \\
\hline
\end{tabular}

Data are presented as mean $\pm \mathrm{SD}$, median (interquartile range) or $\%$ 


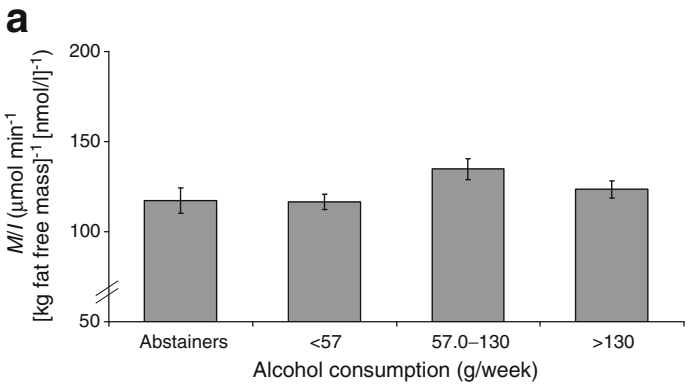

b

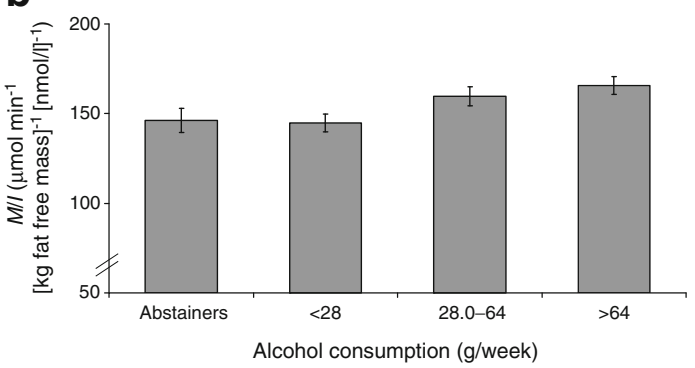

Fig. 1 Insulin sensitivity, expressed as the $M / I$ value according to categories of alcohol consumption (in g/week) in men (a) and women (b). Data are means \pm SEM. $p_{\text {trend }}=0.07$ for men and $p_{\text {trend }}=0.005$ for women, adjusted for age, recruitment centre, physical activity, smoking and waist circumference after a logarithmic transformation for $M / I$

the multivariable model $\left(p_{\text {trend }}=0.02\right)$ and was also independent of fasting glycaemia and fasting insulinaemia.

Impaired glucose tolerance and type 2 diabetes at 3 years Alcohol consumption categories were not associated with either fasting or $2 \mathrm{~h}$ glycaemia at year3 in either men or women, analysed separately in multivariate models. There was a reduced risk of IGT/diabetes at year 3 for the women with a baseline alcohol consumption above the median value ( $>30 \mathrm{~g} /$ week) (OR 0.57, 95\% CI 0.34, 0.94, $p=0.03$ ) after adjusting for age and waist circumference. In men, baseline alcohol consumption above the median value (>71 g/week) was not associated with a reduction in the risk of IGT/diabetes at year3 (OR 1.02, 95\% CI 0.57, $1.81, p=0.95)$.

\section{Discussion}

The main finding of this study is that moderate alcohol consumption is associated with an enhanced peripheral insulin sensitivity, a reduced basal insulin secretion and a lower fasting plasma glucagon concentration in healthy women. This is in agreement with a large body of evidence showing that moderate alcohol consumption is associated with a decreased risk of type 2 diabetes in the general population $[1,7,16-18]$, including women at low risk [6, 19]. A meta-analysis reported a much more marked relation
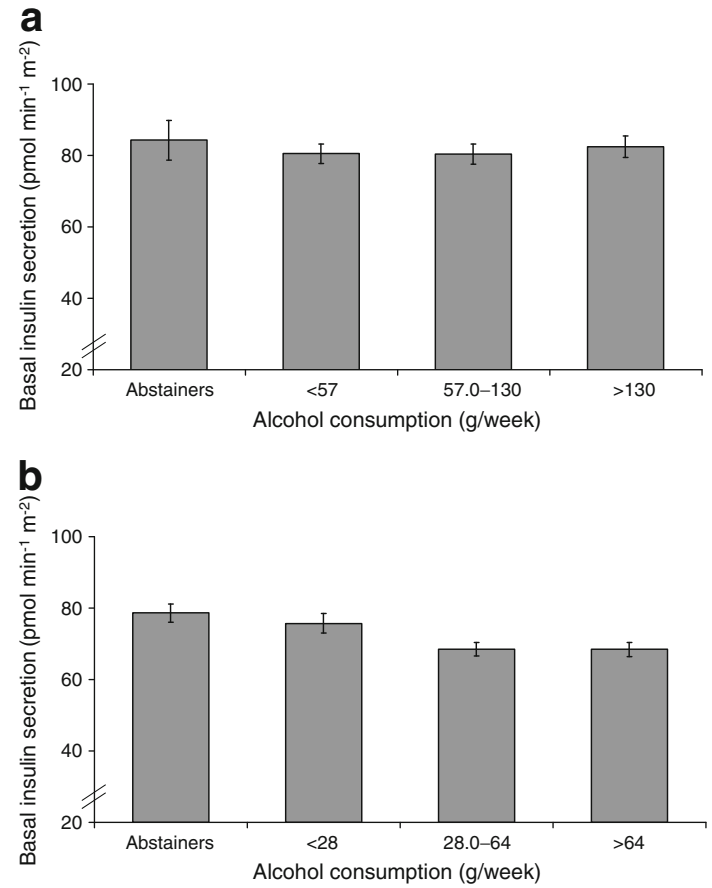

Fig. 2 Basal insulin secretion across alcohol consumption categories in men (a) and women (b). Data are means \pm SEM. $p_{\text {trend }}=0.56$ for men and $p_{\text {trend }}=0.004$ for women, adjusted for age, recruitment centre, physical activity, smoking and waist circumference after a logarithmic transformation for insulin secretion

in women than in men [7]. Similarly, a recent multicentre prospective European study, with 12,403 incident cases of type 2 diabetes and 16,154 controls, showed that moderate alcohol consumption was associated with a reduced risk of type 2 diabetes in women only [20].

Enhanced insulin sensitivity among moderate alcohol drinkers has been reported previously, but most of these studies used proxy estimates of insulin sensitivity, such as the fasting insulin concentration or the HOMA of insulin resistance (HOMA-IR) index, instead of the gold-standard method of the euglycaemic-hyperinsulinaemic clamp, as used in the present study [21-23].

Short-term intervention studies have shown an increase in both insulin sensitivity and adiponectin concentration within 3-8 weeks after alcohol intake [24-27]. Moderate alcohol consumption for 4 weeks has been associated with a $12 \%$ increase in plasma adiponectin and a $57 \%$ increase in its high-molecular-weight form, without concomitant changes in subcutaneous and abdominal fat content or body weight [28]; adiponectin has been shown to account for $25-30 \%$ of the inverse association between alcohol consumption and the risk of type 2 diabetes in women [19]. Interestingly, in the present study, the significant association between moderate alcohol intake and improved insulin sensitivity in women persisted after controlling for adiponectin concentration, suggesting that other mechanisms may be involved. The pathophysiology 
Table 3 Insulin clearance according to categories of alcohol consumption in men. The RISC study

\begin{tabular}{|c|c|c|c|c|c|c|}
\hline \multirow[t]{2}{*}{ Insulin clearance } & \multicolumn{4}{|c|}{ Alcohol consumption (g/week) } & \multirow[t]{2}{*}{$p_{\text {trend }}$} & \multirow[t]{2}{*}{$p_{\text {trend }}$} \\
\hline & Abstainers $(n=70)$ & $<57(n=167)$ & $57-130(n=165)$ & $>130(n=162)$ & & \\
\hline $\begin{array}{l}\text { Peripheral clearance of insulin during the } \\
\text { clamp }\left(1 \mathrm{~min}^{-1} \mathrm{~m}^{-2}\right)\end{array}$ & $0.58 \pm 0.15$ & $0.59 \pm 0.15$ & $0.63 \pm 0.2$ & $0.63 \pm 0.26$ & 0.003 & 0.02 \\
\hline $\begin{array}{l}\text { Endogenous 'pre-hepatic' clearance of insulin } \\
\text { in basal conditions }\left(1 \mathrm{~min}^{-1} \mathrm{~m}^{-2}\right)\end{array}$ & $2.11 \pm 0.71$ & $2.45 \pm 0.94$ & $2.36 \pm 0.78$ & $2.44 \pm 0.78$ & 0.04 & 0.05 \\
\hline $\begin{array}{l}\text { Endogenous 'pre-hepatic' clearance of insulin } \\
\text { during OGTT }\left(1 \mathrm{~min}^{-1} \mathrm{~m}^{-2}\right)\end{array}$ & $1.47 \pm 0.55$ & $1.55 \pm 0.57$ & $1.57 \pm 0.50$ & $1.68 \pm 0.68$ & 0.003 & 0.01 \\
\hline
\end{tabular}

Data are presented as mean $\pm \mathrm{SD}$

$p_{\text {trend }}$ is adjusted for age, centre, physical activity, current smoking, waist circumference

${ }^{a} p_{\text {trend }}$ is additionally adjusted for insulin sensitivity, $\log M / I$

underlying the effects of moderate alcohol intake on insulin sensitivity is complex and not fully understood.

The final product of ethanol oxidation is acetate, which is converted to acetyl-CoA in peripheral tissue [29]. Acetate may modulate fat oxidation and decrease lipolysis. Therefore, an increase in skeletal muscle oxidative capacity after alcohol intake has been suggested even though evidence is limited in humans [28].

HDL-cholesterol concentration increases with alcohol consumption and is also related to insulin sensitivity and adiponectin concentrations, and could be a key mediator in the association between alcohol consumption and improved glucose use [30]. In the present study, the relationship between alcohol intake and HDL-cholesterol levels was stronger in women than in men. Furthermore, the observation that the association between alcohol and insulin sensitivity lost significance after further adjustment for HDLcholesterol concentration supports a greater role for HDLcholesterol, rather than for adiponectin, in the modulation of insulin sensitivity by alcohol, in particular among women.

The effects of alcohol consumption on insulin secretion have been investigated less extensively than its effects on insulin sensitivity, in particular with a conjoint assessment of insulin sensitivity. In the present study, we found that light-to-moderate alcohol consumption was associated with a decreased basal insulin secretion rate in non-diabetic women, and this effect was independent of insulin sensitivity. This finding was confirmed when we assessed basal insulin secretion at a fixed normal glucose level of $5 \mathrm{mmol} / \mathrm{l}$ glucose from the beta cell dose-response. These results suggest that alcohol has a more pronounced effect on basal insulin secretion rate than on glucose-load-induced insulin response. Data on the effects of alcohol consumption on insulin secretion are conflicting: some studies show the absence of an association in men [31,32] and others show a decreased insulin secretion with men and women combined [17]. The reasons for the absence of a significant association between alcohol intake and insulin secretion in men in contrast to women remain unclear, and may rely on sex specificity. Adjustment for menopausal status did not substantially modify the association between alcohol consumption and either insulin sensitivity or secretion (data not shown), suggesting that sex-specific effects may be related to effects other than differences in hormone levels.

In agreement with our findings, an inverse association between alcohol intake and insulin secretion has been noted

Table 4 Insulin clearance according to categories of alcohol consumption in women. The RISC study

\begin{tabular}{|c|c|c|c|c|c|c|}
\hline \multirow[t]{2}{*}{ Insulin clearance } & \multicolumn{4}{|c|}{ Alcohol consumption (g/week) } & \multirow[t]{2}{*}{$p_{\text {trend }}$} & \multirow[t]{2}{*}{$p_{\text {trend }}$} \\
\hline & Abstainers $(n=171)$ & $<28(n=166)$ & $28-64(n=181)$ & $>64(n=184)$ & & \\
\hline $\begin{array}{l}\text { Peripheral clearance of insulin during the } \\
\text { clamp }\left(1 \mathrm{~min}^{-1} \mathrm{~m}^{-2}\right)\end{array}$ & $0.62 \pm 0.41$ & $0.63 \pm 0.19$ & $0.63 \pm 0.13$ & $0.64 \pm 0.22$ & 0.04 & 0.49 \\
\hline $\begin{array}{l}\text { Endogenous 'pre-hepatic' clearance of insulin } \\
\text { in basal conditions }\left(1 \mathrm{~min}^{-1} \mathrm{~m}^{-2}\right)\end{array}$ & $2.28 \pm 0.76$ & $2.29 \pm 0.72$ & $2.43 \pm 0.81$ & $2.53 \pm 0.80$ & 0.22 & 0.53 \\
\hline $\begin{array}{l}\text { Endogenous 'pre-hepatic' clearance of insulin } \\
\text { during OGTT }\left(1 \mathrm{~min}^{-1} \mathrm{~m}^{-2}\right)\end{array}$ & $1.62 \pm 0.60$ & $1.61 \pm 0.56$ & $1.71 \pm 0.61$ & $1.74 \pm 0.54$ & 0.28 & 0.91 \\
\hline
\end{tabular}

Data are presented as mean $\pm \mathrm{SD}$

$p_{\text {trend }}$ is adjusted for age, centre, physical activity, current smoking, waist circumference

${ }^{a} p_{\text {trend }}$ is additionally adjusted for insulin sensitivity, $\log M / I$ 

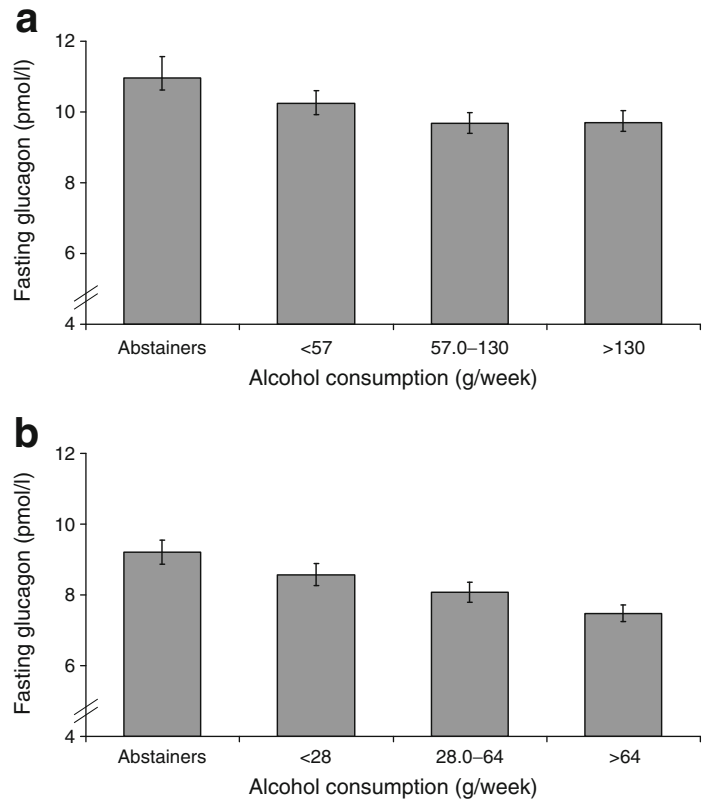

Fig. 3 Fasting glucagon concentration according to categories of alcohol consumption (in $\mathrm{g} / \mathrm{week}$ ) in men (a) and women (b). Data are means \pm SEM. $p_{\text {trend }}=0.37$ for men and $p_{\text {trend }}=0.01$ for women, adjusted for age, recruitment centre, physical activity, smoking and waist circumference after a logarithmic transformation for glucagon

previously in non-diabetic women [33]. The decrease in basal insulin secretion rate across alcohol consumption categories in women could be viewed as a direct consequence of enhanced insulin sensitivity. Furthermore, exposure to physiological hyperinsulinaemia stimulates insulin secretion in non-diabetic individuals [14]. However, adjustment for insulin sensitivity did not alter the inverse association, suggesting alternative mechanisms. Furthermore, the effect of alcohol on insulin secretion appears to be independent of the concomitant increase in either HDL-cholesterol or adiponectin concentrations. Therefore, the possibility of a specific effect of alcohol on beta cell function needs to be further explored.

The present study showed that alcohol consumption was significantly associated with an increased endogenous clearance of insulin. This aspect has not previously been recognised. Enhanced rates of insulin clearance across alcohol intake categories may be related to concomitant increased insulin sensitivity and reduced basal insulin secretion rate as seen in the present study. Previous studies have underscored the tight interactions between insulin sensitivity, insulin secretion and insulin clearance [34-36]. In the presence of insulin resistance, glucose homeostasis is maintained by an increase in plasma insulin via increased secretion and a decrease in insulin extraction. Animal studies have shown that induction of insulin resistance induces an initial increase in insulin secretion followed by a decrease in hepatic insulin extraction [37].
The graded increase in NEFA concentration across alcohol consumption categories observed in the present study is consistent with both the increased clearance of insulin and reduced basal insulin secretion rate, observed in parallel with increasing alcohol intake, as insulin inhibits lipolysis and NEFA release in healthy individuals [38].

To our knowledge, the relationship between alcohol and glucagon concentration has not been investigated previously. Our finding of an inverse correlation between alcohol consumption and fasting glucagon levels in women suggests a putative new mechanism to explain why moderate alcohol consumption is protective for the development of type 2 diabetes. A direct effect of alcohol on the regulation of glucagon release warrants specific investigation. This inverse association between glucagon and alcohol consumption appeared to be independent of fasting glycaemia and of insulinaemia, suggesting a specific, yet poorly characterised, direct effect of alcohol on the regulation of glucagon release. This novel finding of an inverse correlation between moderate alcohol consumption and fasting glucagon concentrations should also be tested in type 2 diabetes.

Limitations of the present study include its crosssectional design, which precludes drawing causal inferences from the observed associations. Furthermore, our results may have been influenced by differences in factors other than alcohol intake. We cannot rule out confounding by unmeasured variables. The difficulty of an accurate evaluation of alcohol consumption with possible under-reporting and misclassification should be acknowledged. However, we intentionally excluded heavy drinkers in our study to focus on abstainers and light-to-moderate drinkers.

The strengths of the present study are the large RISC cohort of healthy participants, the use of the gold-standard methodology for measurement of insulin sensitivity, use of centralised laboratory assays with a quality control of data and the assessment of both insulin secretion and insulin clearance [8]. This provided the opportunity to systematically explore, in both men and women, the relationship between alcohol consumption and insulin sensitivity, insulin secretion, insulin clearance and glucagon concentrations.

In conclusion, our study shows that in healthy women, light-to-moderate alcohol consumption is associated with enhanced insulin sensitivity, decreased basal insulin secretion rate, decreased fasting glucagon levels and an increased insulin clearance. These findings provide potential explanations for the reduced risk of type 2 diabetes associated with moderate alcohol consumption.

Funding The RISC study was supported by EU grant QLG1-CT2001-01252, with additional support from AstraZeneca (Sweden). The European Group for the Study of Insulin Resistance (EGIR) group activities are supported by an unrestricted research grant from Merck Serono, France. 
Duality of interest The authors declare that there is no duality of interest associated with this manuscript.

Contribution statement FB was responsible for the conception of the study, analysed data and wrote the manuscript, ED, ML, AM, KH, CHA, PP and BB analysed data and revised the manuscript. All authors approved the final version.

\section{References}

1. Koppes LL, Dekker JM, Hendriks HF, Bouter LM, Heine RJ (2005) Moderate alcohol consumption lowers the risk of type 2 diabetes: a meta-analysis of prospective observational studies. Diabetes Care 28:719-725

2. Beulens JW, Stolk RP, van der Schouw YT, Grobbee DE, Hendriks HF, Bots ML (2005) Alcohol consumption and risk of type 2 diabetes among older women. Diabetes Care 28:2933-2938

3. Conigrave KM, Hu BF, Camargo CA Jr, Stampfer MJ, Willett WC, Rimm EB (2001) A prospective study of drinking patterns in relation to risk of type 2 diabetes among men. Diabetes 50:2390-2395

4. Djousse L, Biggs ML, Mukamal KJ, Siscovick DS (2007) Alcohol consumption and type 2 diabetes among older adults: the Cardiovascular Health Study. Obesity (Silver Spring) 15:1758-1765

5. Hodge AM, English DR, O’Dea K, Giles GG (2006) Alcohol intake, consumption pattern and beverage type, and the risk of type 2 diabetes. Diabetic Medicine: A Journal of the British Diabetic Association 23:690-697

6. Wannamethee SG, Camargo CA Jr, Manson JE, Willett WC, Rimm EB (2003) Alcohol drinking patterns and risk of type 2 diabetes mellitus among younger women. Arch Intern Med 163:1329-1336

7. Baliunas DO, Taylor BJ, Irving H et al (2009) Alcohol as a risk factor for type 2 diabetes: a systematic review and meta-analysis. Diabetes Care 32:2123-2132

8. Hills SA, Balkau B, Coppack SW et al (2004) The EGIR-RISC STUDY (The European group for the study of insulin resistance: relationship between insulin sensitivity and cardiovascular disease risk): I. Methodology and objectives. Diabetologia 47:566-570

9. Bonnet F, Patel S, Laville M et al (2008) Influence of the ACE gene insertion/deletion polymorphism on insulin sensitivity and impaired glucose tolerance in healthy subjects. Diabetes Care 31:789-794

10. Hallal PC, Victora CG (2004) Reliability and validity of the International Physical Activity Questionnaire (IPAQ). Med Sci Sports Exerc 36:556

11. Frystyk J, Tarnow L, Hansen TK, Parving HH, Flyvbjerg A (2005) Increased serum adiponectin levels in type 1 diabetic patients with microvascular complications. Diabetologia 48:1911-1918

12. Van Cauter E, Mestrez F, Sturis J, Polonsky KS (1992) Estimation of insulin secretion rates from C-peptide levels. Comparison of individual and standard kinetic parameters for C-peptide clearance. Diabetes 41:368-377

13. Mari A, Schmitz O, Gastaldelli A, Oestergaard T, Nyholm B, Ferrannini E (2002) Meal and oral glucose tests for assessment of beta-cell function: modeling analysis in normal subjects. Am J Physiol Endocrinol Metabol 283:E1159-E1166

14. Mari A, Tura A, Natali A et al (2011) Influence of hyperinsulinemia and insulin resistance on in vivo beta-cell function: their role in human beta-cell dysfunction. Diabetes 60:3141-3147

15. Mari A, Tura A, Natali A et al (2010) Impaired beta cell glucose sensitivity rather than inadequate compensation for insulin resistance is the dominant defect in glucose intolerance. Diabetologia 53:749-756
16. Ajani UA, Hennekens CH, Spelsberg A, Manson JE (2000) Alcohol consumption and risk of type 2 diabetes mellitus among US male physicians. Arch Intern Med 160:1025-1030

17. Crandall JP, Polsky S, Howard AA et al (2009) Alcohol consumption and diabetes risk in the Diabetes Prevention Program. Am J Clin Nutr 90:595-601

18. Joosten MM, Chiuve SE, Mukamal KJ, Hu FB, Hendriks HF, Rimm EB (2011) Changes in alcohol consumption and subsequent risk of type 2 diabetes in men. Diabetes 60:74-79

19. Beulens JW, Rimm EB, Hu FB, Hendriks HF, Mukamal KJ (2008) Alcohol consumption, mediating biomarkers, and risk of type 2 diabetes among middle-aged women. Diabetes Care 31:2050-2055

20. Beulens JW, van der Schouw YT, Bergmann MM et al (2012) Alcohol consumption and risk of type 2 diabetes in European men and women: influence of beverage type and body size. The EPIC-InterAct study. J Intern Med Feb 21. doi:10.1111/j.1365-2796.2012.02532.x

21. Lazarus R, Sparrow D, Weiss ST (1997) Alcohol intake and insulin levels. The normative aging study. Am J Epidemiol 145:909-916

22. Kiechl S, Willeit J, Poewe W et al (1996) Insulin sensitivity and regular alcohol consumption: large, prospective, cross sectional population study (Bruneck study). BMJ 313:1040-1044

23. Kroenke $\mathrm{CH}$, Chu NF, Rifai $\mathrm{N}$ et al (2003) A cross-sectional study of alcohol consumption patterns and biologic markers of glycemic control among 459 women. Diabetes Care 26:1971-1978

24. Davies MJ, Baer DJ, Judd JT, Brown ED, Campbell WS, Taylor PR (2002) Effects of moderate alcohol intake on fasting insulin and glucose concentrations and insulin sensitivity in postmenopausal women: a randomized controlled trial. JAMA 287:2559-2562

25. Sierksma A, Patel H, Ouchi N et al (2004) Effect of moderate alcohol consumption on adiponectin, tumor necrosis factor-alpha, and insulin sensitivity. Diabetes Care 27:184-189

26. Beulens JW, van Beers RM, Stolk RP, Schaafsma G, Hendriks HF (2006) The effect of moderate alcohol consumption on fat distribution and adipocytokines. Obesity (Silver Spring) 14:60-66

27. Joosten MM, Beulens JW, Kersten S, Hendriks HF (2008) Moderate alcohol consumption increases insulin sensitivity and ADIPOQ expression in postmenopausal women: a randomised, crossover trial. Diabetologia 51:1375-1381

28. Beulens JW, van Loon LJ, Kok FJ et al (2007) The effect of moderate alcohol consumption on adiponectin oligomers and muscle oxidative capacity: a human intervention study. Diabetologia 50:1388-1392

29. Siler SQ, Neese RA, Hellerstein MK (1999) de novo lipogenesis, lipid kinetics, and whole-body lipid balances in humans after acute alcohol consumption. Am J Clin Nutr 70:928-936

30. Drew BG, Duffy SJ, Formosa MF et al (2009) High-density lipoprotein modulates glucose metabolism in patients with type 2 diabetes mellitus. Circulation 119:2103-2111

31. Avogaro A, Watanabe RM, Gottardo L, de Kreutzenberg S, Tiengo A, Pacini G (2002) Glucose tolerance during moderate alcohol intake: insights on insulin action from glucose/lactate dynamics. J Clin Endocrinol Metab 87:1233-1238

32. Riserus U, Ingelsson E (2007) Alcohol intake, insulin resistance, and abdominal obesity in elderly men. Obesity (Silver Spring) 15:1766-1773

33. Greenfield JR, Samaras K, Jenkins AB, Kelly PJ, Spector TD, Campbell LV (2003) Moderate alcohol consumption, estrogen replacement therapy, and physical activity are associated with increased insulin sensitivity: is abdominal adiposity the mediator? Diabetes Care 26:2734-2740

34. Ferrannini E, Wahren J, Faber OK, Felig P, Binder C, DeFronzo RA (1983) Splanchnic and renal metabolism of insulin in human subjects: a dose-response study. Am J Physiol 244:E517-E527

35. Meier JJ, Veldhuis JD, Butler PC (2005) Pulsatile insulin secretion dictates systemic insulin delivery by regulating hepatic insulin extraction in humans. Diabetes 54:1649-1656 
36. Tillil H, Shapiro ET, Rubenstein AH, Galloway JA, Polonsky KS (1988) Reduction of insulin clearance during hyperglycemic clamp. Dose-response study in normal humans. Diabetes 37:13511357

37. Kim SP, Ellmerer M, Kirkman EL, Bergman RN (2007) Beta-cell "rest" accompanies reduced first-pass hepatic insulin extraction in the insulin-resistant, fat-fed canine model. Am J Physiol Endocrinol Metabol 292:E1581-E1589

38. Groop LC, Bonadonna RC, DelPrato S et al (1989) Glucose and free fatty acid metabolism in non-insulin-dependent diabetes mellitus. Evidence for multiple sites of insulin resistance. J Clin Invest 84:205-213 